\title{
IJAHSP:-
}

The Internet Joưnal of Allied Health Sciences and Practice

A Peer Reviewed Publication of the College of Health Care Sciences at Nova Southeastern University

Dedicated to allied health professional practice and education

http://ijahsp.nova.edu Vol. 11 No. ISSN 1540-580X

\section{Clinical Academic Faculty Perceptions of Academic Mentorship in the Health Professions}

\author{
Leamor Kahanov, EdD, ATC1 \\ Lindsey Eberman, PhD, ATC2 \\ Tiffany Idlewine, $\mathrm{DPT}^{3}$ \\ Lynda Melton, PA-ARC ${ }^{3}$
}

1. Chair and Professor, Department of Applied Medicine and Rehabilitation, Indiana State University, Terre Haute, Indiana

2. Program Director and Associate Professor, Department of Applied Medicine and Rehabilitation, Indiana State University, Terre Haute, Indiana

3. Assistant Professor, Department of Applied Medicine and Rehabilitation, Indiana State University, Terre Haute, Indiana

United States

CITATION: Kahanov L, Eberman L, Idlewine T, Melton L. Clinical Academic Faculty Perceptions of Academic Mentorship in the Health Professions. The Internet Journal of Allied Health Sciences and Practice. July 2013. Volume 11 Number 4.

\section{ABSTRACT}

Mentorship in the first three years of academic employment is pivotal for retention, productivity, and overall satisfaction and success of academic faculty members. We used an observational, descriptive research design with a web-based survey to evaluate perceived knowledge and perceptions of mentorship among health care professional academic faculty (PT, PA, OT, and AT) nationwide. Academic faculty that indicated they experienced mentorship consistently reported positive outcomes. Respondents described mentorship experiences as both positive and negative dependent upon the mentor. Respondents indicated that mentors assisted with publications, presentations/posters, new teaching methods/strategies, clinical expertise, conducting research, service activities, community engagement activities, program development, job change/promotion, and grant writing/submissions. Assessment of pre and post-mentorship perceived knowledge of academic faculty requirements indicated significant differences. Most respondents indicated little knowledge of academic faculty expectations pre mentorship and significant increases in knowledge post mentorship. Concerns are likely due to the incongruity between expectations articulated (or not articulated) during doctoral studies or clinical experiences and the actual requirements of a full-time academic faculty member. Mentored academic faculty had more positive experiences and demonstrated a better understanding of scholarship and teaching. However, a lack of understanding regarding institutional hierarchy/policies and service/community engagement are areas of mentorship need and may contribute to faculty mismatched expectations, negative retention, and clinical academic faculty perceived success.

\section{INTRODUCTION}

The first few years in academia can be challenging and stressful. ${ }^{1,2}$ These challenges have led to as much as $82 \%$ of new academic faculty in the United States (known as academic staff in other countries) seeking other institutions for employment in their first year. ${ }^{3}$ New academic faculty who have not undergone traditional doctoral training may be subject to additional challenges. ${ }^{4-6}$ Accompanying the proliferation of clinical doctorate degrees/terminal degrees in the last 15 years has been the hiring of clinically trained individuals as tenure-track academic faculty to help support the curricular needs of programs. ${ }^{7-15}$ Clinically trained academic faculty may not be oriented to academic expectations regarding scholarship, teaching, and service set forth by the institution and accrediting bodies, placing them at a disadvantage.3,8, 12 
Mentorship in the first three years of academic employment is pivotal for the future satisfaction and success of academic faculty members. ${ }^{1}$ Junior academic faculty face the challenges of budgeting time effectively, understanding academic expectations, and developing positive relationships with colleagues. ${ }^{12} \mathrm{New}$ academic faculty often have difficulty transitioning, in large part because of incongruity between the expectations, during doctoral studies and the actual requirements of a full-time academic faculty member. ${ }^{5}$

Challenges in socialization are compounded with clinically trained doctoral academic faculty who lack the foundational orientation to academia that traditionally trained doctoral academic faculty obtain during their advanced studies. The incongruity between clinical doctorate education, which focuses on creating clinicians, and the requirements of universities, which often defer to individualized (institutional or departmental) tenure and promotion guidelines, complicates the transition from clinical practice to academic faculty. Academic faculty transitioning from the clinical setting to academia may need additional orientation to institutional hierarchy and tenure and promotion requirements such as scholarship, service, and teaching. A comprehensive understanding of the needs of clinicians transitioning to academic faculty are not well understood. Research on academic faculty mentorship has focused primarily on traditionally trained doctoral academic faculty. The purpose of this research study was to survey clinically trained academic faculty members in healthcare professions to gain an understanding of their perceived knowledge of academia prior to becoming an academic faculty member, the level of mentorship received once in an academic faculty position, and the perceptions of the mentorship experience.

\section{METHODS}

\section{Research Design}

An observational, descriptive research design with a web-based survey (www.surveymonkey.com) was used to evaluate perceived knowledge and perceptions of mentorship among health care professional academic faculty.

\section{Participants}

All academic faculty listed on public websites for PT, PA, OT and AT were contacted with a response rate of $31 \%(493 / 1603)$. It was chosen not to query academic faculty from physician medical education programs/schools and focus on health professions. Total respondents both clinical faculty and tenure/tenure track were physical therapy (PT, $n=3)$, physician assistant (PA, $n=173$ ), occupational therapy (OT, n=112), athletic training ( $A T, n=203)$ and two undisclosed. Our target were academic faculty with a terminal clinical practice degree and thus academic faculty with a traditional terminal degree or who were never mentored indicated such in the preliminary aspects of the survey and were guided to the end of the survey $(n=244 ; P h D=155, E d D=50$, other terminal degree=39). Clinical faculty respondents constituted $16 \%(249 / 1603)$ of the population surveyed with 106 athletic training, 36 occupational therapy, 106 physician assistant clinical faculty and one undisclosed. We contacted potential participants in October, which allowed them to reflect on their mentorship experiences if they had just started a new position. The Indiana State University Institutional Review Board awarded human subjects approval before data were collected. Consent to participate in study was implied by responding to the survey. All respondents were both anonymous and confidential through use of an online survey tool that prohibits IP and email address recognition.

\section{Instruments}

A web-based survey was disseminated through www.surveymonkey.com to acquire anonymous data from our participants. We adapted a survey used in previous research by Smith.,10 Academic faculty perceptions of mentor selection, mentor encounters, mentoring characteristics, mentorship benefits, and the knowledge level of academic faculty requirements prior to mentorship and post mentorship were collected. The instrument yielded strong internal consistency throughout (Cronbach's alpha=0.958).

The initial section of the survey collected data on the type of terminal clinical degree the academic faculty member earned (i.e. AT, OT, PT, PA), years in clinical practice, gender, terminal degree, years in academia, and type of position in academia. Academic faculty members rated their knowledge level of a multitude of academic responsibilities prior to beginning their academic position. Then respondents articulated the mentor selection process, the frequency of mentor encounters and the information exchanged during mentor encounters. The participants were then asked to describe their perceived knowledge of the same academic responsibilities after mentorship. The last section of the survey provided a list of benefits respondents indicated may have resulted from the mentorship experience.

\section{Procedures}

Contact information for PT, PA, OT, and AT academic faculty were acquired nationwide using publically accessible university websites. All the email addresses were entered into the web-based survey and disseminated an initial email. After 1 week, a follow-up email was sent to elicit additional responses and the survey remained open for a total of 3 weeks. 


\section{Data Analysis}

The data were analyzed for descriptive statistics using SPSS 18.0. Pre and post-mentorship perceived knowledge using paired samples t-tests were performed. Further, participants were grouped by mentor selection/assignment, a categorical variable, and compared for their post-mentorship perceptions using separate Kruskal-Wallis non-parametric one-way ANOVAs. The a-priori alpha level was set at $p<0.05$ for the inferential statistics using non-parametric statistics to account for unequal group sizes in the mentor selection/assignments analyses.

\section{RESULTS}

\section{Demographics}

The majority of respondents were ATs $(n=203)$, OTs $(n=112)$, PAs $(n=173)$, and PTs $(n=3)$ with two undeclared respondents. The majority of respondents were female $(n=309,62.8 \%$; male $=184,37.2 \%)$ and tenured/tenure track academic faculty $(n=244$, 49.6\%; clinical academic faculty=81, 16.4\%; instructor/full-time non-tenure track=107, 21.8\%; Lecturer/Part-time=22, 4.4\%; and Other $=39,7.8 \%)$. Although several academic faculty were eliminated as traditionally trained academic faculty $(n=205 ; \mathrm{PhD}=155$, $\mathrm{EdD}=50)$, over half of the respondents met the criteria of clinical doctorate/terminal degree academic faculty DPT $(n=2,0.4 \%)$; DOT $(n=8,1.6 \%)$; DSc $(n=12,2.5 \%)$; MPAS/MSPA $(n=63,12.9 \%)$ MS/MA $(n=19840.2 \%$,$) and 9(1.8 \%)$ undisclosed. A majority of respondents indicated not having a designated mentor $(n=164,66.1 \%)$ while others were assigned or selected mentors (Table 1).

Table 1a. Mentor Selection/Assignment Process ( $\mathrm{n}=243$ responses)

\begin{tabular}{|l|l|l|}
\hline Selection/Assignment & $\mathbf{N}$ & $\%$ \\
\hline Assigned a mentor & 40 & $16.5 \%$ \\
\hline Assign and selected mentors & 10 & $4.1 \%$ \\
\hline Selected a mentor & 15 & $6.2 \%$ \\
\hline Selected several mentors & 18 & $7.4 \%$ \\
\hline Did not have a designated mentor & 160 & $65.8 \%$ \\
\hline Did not desire a mentor & 0 & $0.0 \%$ \\
\hline
\end{tabular}

Table 1b. Mentor Selection/Assignment Categories for Statistical Grouping in Post-Mentorship Analysis ( $\mathrm{n}=83$ responses)

\begin{tabular}{|l|l|l|}
\hline Selection/Assignment & N & $\%$ \\
\hline Assigned a mentor & 40 & $48.2 \%$ \\
\hline Assign and selected mentors & 10 & $12.0 \%$ \\
\hline Selected a/several mentors & 33 & $39.8 \%$ \\
\hline
\end{tabular}

\section{Mentorship Experiences}

Academic faculty that indicated they experienced mentorship during academic employment $(n=82)$ consistently reported positive outcomes. A majority of respondents consistently indicated that their mentors were helpful in a variety of ways (Table 2); however, mentors were not particularly helpful in aiding respondents in understanding the tenure and promotion process (mean \pm SD) or preparing tenure and promotion submissions for review (mean \pm SD), or suggesting appropriate resources (MeanSD).

Table 2. Mentorship Experiences (Mean Scores and \% Majority Responses)

\begin{tabular}{|c|c|c|c|}
\hline & Mean & \pm SD & \% Majority Responses \\
\hline My mentor was accessible & 5.79 & 1.59 & $S T A=32,39.0 \%$ \\
\hline My mentor demonstrated professional integrity & 6.09 & 1.83 & STA $=44,53.7 \%$ \\
\hline My mentor demonstrated content expertise in my area of need & 5.27 & 2.25 & STA $=36,43.9 \%$ \\
\hline My mentor was approachable & 5.90 & 2.19 & STA $=50,61.0 \%$ \\
\hline My mentor was supportive and encouraging & 5.87 & 2.00 & STA $=61,62.2 \%$ \\
\hline My mentor provided constructive and useful critiques of my work & 5.41 & 2.08 & $A=32,39.0 \%$ \\
\hline My mentor was helpful in providing direction and guidance on professional issues & 5.39 & 1.84 & STA $=29,35.4 \%$ \\
\hline My mentor answered my questions satisfactorily & 5.74 & 1.51 & $A=33,40.2 \%$ \\
\hline My mentor acknowledge my contributions appropriately & 5.40 & 1.65 & $A=30,36.6 \%$ \\
\hline My mentor aided me in understanding tenure and promotion expectations & 5.01 & 1.86 & NAD $=21,25.9 \%$ \\
\hline My mentor aided me in preparing my tenure and promotion submission & 4.57 & 1.93 & $\mathrm{NAD}=26,32.9 \%$ \\
\hline My mentor suggested appropriate resources & 3.52 & 2.10 & $S T A=23,28.0 \%, A=23,28.0 \%$ \\
\hline My mentor challenged me to extend my abilities & 5.13 & 1.84 & STA $=24,29.3 \%$ \\
\hline
\end{tabular}

7-point Likert scale: STA (7)=Strongly Agree, A (6)=Agree, SLA (5)=Slightly Agree, NAD (4)=Neither Agree or Disagree, SLD (3)=Slightly

Disagree, D (2)=Disagree, STD (1)=Strongly Disagree 


\section{Perceived Knowledge of Academic Faculty Requirements}

Pre and post-mentorship perceived knowledge of academic faculty requirements were analyzed and significant differences were found (Table 3). Most respondents indicated little knowledge at all regarding academic faculty expectations. After mentorship, these respondents reported significant increases in knowledge with the exception of 6 categories that represent academic faculty requirements in teaching, grant writing, and institutional knowledge.

Also compared were perceived knowledge of academic faculty requirements depending on how mentors were selected/assigned to demonstrate differences between those that were assigned versus those that were selected or selected and assigned mentors (Table 1b). A significant difference was found between groups (Table 4) that selected their mentors or the combination of selected and assigned mentors versus mentor assignment alone.

Table 3. Significant Differences Between Pre and Post-Mentorship on Perceived Knowledge of Academic Faculty Responsibilities

\begin{tabular}{|c|c|c|c|c|}
\hline Academic Faculty Responsibilities & $\begin{array}{l}\text { Pre-Mentorship } \\
\text { (Mean } \pm S D \text { ) }\end{array}$ & $\begin{array}{l}\text { Post-Mentorship } \\
\text { (Mean } \pm \text { SD) }\end{array}$ & Paired t-test & $p$ value \\
\hline Research agenda* & $2.37 \pm 0.98$ & $3.15 \pm 0.94$ & $t_{61}=-5.913$ & $p<0.001$ \\
\hline How to conduct research* & $2.42 \pm 0.82$ & $2.95 \pm 0.85$ & $t_{65}=-4.891$ & $p<0.001$ \\
\hline Publication requirements* & $2.44 \pm 0.96$ & $3.24 \pm 1.03$ & $t_{69}=-6.782$ & $p<0.001$ \\
\hline Presentations (local/national) ${ }^{*}$ & $2.74 \pm 1.00$ & $3.62 \pm 0.98$ & $t_{67}=-6.311$ & $p<0.001$ \\
\hline Evaluation techniques* & $2.77 \pm 1.12$ & $3.51 \pm 1.03$ & $t_{69}=-5.461$ & $p<0.001$ \\
\hline Program assessment ${ }^{*}$ & $2.38 \pm 1.11$ & $3.36 \pm 1.15$ & $t_{68}=-6.588$ & $p<0.001$ \\
\hline Teaching philosophy & $3.67 \pm 1.50$ & $3.33 \pm 1.03$ & $t_{69}=1.758$ & $p=0.083$ \\
\hline Teaching methods* & $4.26 \pm 0.86$ & $3.46 \pm 1.02$ & $t_{69}=4.463$ & $p<0.001$ \\
\hline Institutional hierarchy* & $4.23 \pm 0.90$ & $3.34 \pm 0.99$ & $t_{69}=5.219$ & $p<0.001$ \\
\hline $\begin{array}{l}\text { Service requirements (community, } \\
\text { profession, etc.) }\end{array}$ & $4.14 \pm 0.92$ & $3.39 \pm 0.95$ & $t_{69}=4.372$ & $p<0.001$ \\
\hline $\begin{array}{l}\text { Institutional service requirements } \\
\text { (committees, etc.) }\end{array}$ & $3.50 \pm 1.57$ & $3.34 \pm 0.95$ & $t_{69}=0.712$ & $p=0.479$ \\
\hline Grant writing & $2.77 \pm 1.87$ & $2.44 \pm 1.03$ & $t_{69}=1.556$ & $p=0.124$ \\
\hline Grant submission* & $1.69 \pm 0.84$ & $2.44 \pm 1.06$ & $t_{69}=-6.435$ & $p<0.001$ \\
\hline Advising* $^{*}$ & $2.49 \pm 1.05$ & $3.56 \pm 0.99$ & $t_{69}=-8.094$ & $p<0.001$ \\
\hline Time commitment* & $2.80 \pm 1.11$ & $3.84 \pm 0.79$ & $t_{69}=-7.603$ & $p<0.001$ \\
\hline Community engagement* & $2.66 \pm 0.98$ & $3.47 \pm 0.97$ & $t_{69}=-5.381$ & $p<0.001$ \\
\hline Service learning* $^{*}$ & $2.66 \pm 0.98$ & $3.57 \pm 1.00$ & $t_{69}=-5.965$ & $p<0.001$ \\
\hline Student professional guidance* & $2.60 \pm 1.02$ & $3.65 \pm 0.84$ & $\mathrm{t}_{67}=-7.650$ & $p<0.001$ \\
\hline Clinical and educational contracts* & $2.38 \pm 1.16$ & $3.23 \pm 1.13$ & $t_{68}=-6.446$ & $p<0.001$ \\
\hline Creating a course ${ }^{*}$ & $2.33 \pm 0.99$ & $3.52 \pm 0.96$ & $t_{63}=-9.444$ & $p<0.001$ \\
\hline Creating syllabi* & $2.36 \pm 1.13$ & $3.63 \pm 0.98$ & $t_{69}=-9.322$ & $p<0.001$ \\
\hline Course/Curricular sequencing* & $2.26 \pm 0.99$ & $3.43 \pm 1.00$ & $\mathrm{t}_{67}=-8.036$ & $p<0.001$ \\
\hline Socialization to academic culture* & $2.12 \pm 0.92$ & $3.51 \pm 0.96$ & $\mathrm{t}_{68}=-9.705$ & $p<0.001$ \\
\hline $\begin{array}{l}\text { Expectations of institutional social } \\
\text { responsibilities* }^{*}\end{array}$ & $2.14 \pm 0.91$ & $3.30 \pm 1.02$ & $t_{68}=-7.972$ & $p<0.001$ \\
\hline Collegiality and civility* & $2.95 \pm 1.04$ & $3.56 \pm 1.08$ & $\mathrm{t}_{65}=-4.971$ & $p<0.001$ \\
\hline
\end{tabular}

5 -point Likert scale: $5=$ great knowledge, $4=$ =more than average knowledge, $3=$ =average knowledge, $2=$ little knowledge, $1=$ no knowledge at all

${ }^{*}$ Significance differences at $p<0.001$ 
Table 4. Significant Differences Between Mentor Selection/Assignment Categories and Post-Mentorship Perceived Knowledge of Academic Faculty Responsibilities

\begin{tabular}{|c|c|c|c|c|}
\hline Academic Faculty Responsibilities & $\begin{array}{l}\text { One-Way ANOVA } \\
\text { (K-W test) }\end{array}$ & $p$ & $\begin{array}{l}\text { Mentor Selection/ } \\
\text { Assignment Classification }\end{array}$ & Mean \pm SD \\
\hline \multirow[t]{3}{*}{ Research agenda* } & \multirow[t]{3}{*}{$x^{2}=7.270(d f=2)$} & \multirow[t]{3}{*}{$p=0.026$} & Assigned & $2.94 \pm 0.85$ \\
\hline & & & Assigned and Selected & $3.67 \pm 1.00$ \\
\hline & & & Selected & $3.50 \pm 0.76$ \\
\hline \multirow[t]{3}{*}{ How to conduct research } & \multirow[t]{3}{*}{$X^{2}=3.104(d f=2)$} & \multirow[t]{3}{*}{$p=0.212$} & Assigned & $2.85 \pm 0.87$ \\
\hline & & & Assigned and Selected & $3.22 \pm 1.30$ \\
\hline & & & Selected & $3.27 \pm 0.83$ \\
\hline \multirow[t]{3}{*}{ Publication requirements } & \multirow[t]{3}{*}{$x^{2}=4.172(d f=2)$} & \multirow[t]{3}{*}{$p=0.124$} & Assigned & $3.06 \pm 0.92$ \\
\hline & & & Assigned and Selected & $3.67 \pm 1.32$ \\
\hline & & & Selected & $3.42 \pm 0.95$ \\
\hline \multirow[t]{3}{*}{ Presentations (local/national)* } & \multirow[t]{3}{*}{$x^{2}=9.241(d f=2)$} & \multirow[t]{3}{*}{$p=0.010$} & Assigned & $3.24 \pm 0.96$ \\
\hline & & & Assigned and Selected & $3.89 \pm 1.05$ \\
\hline & & & Selected & $4.00 \pm 0.80$ \\
\hline \multirow[t]{3}{*}{ Evaluation techniques } & \multirow[t]{3}{*}{$x^{2}=3.830(d f=2)$} & \multirow[t]{3}{*}{$p=0.147$} & Assigned & $3.29 \pm 1.09$ \\
\hline & & & Assigned and Selected & $3.57 \pm 1.01$ \\
\hline & & & Selected & $3.85 \pm 0.88$ \\
\hline \multirow[t]{3}{*}{ Program assessment } & \multirow[t]{3}{*}{$x^{2}=3.432(d f=2)$} & \multirow[t]{3}{*}{$p=0.180$} & Assigned & $3.18 \pm 1.24$ \\
\hline & & & Assigned and Selected & $3.00 \pm 1.23$ \\
\hline & & & Selected & $3.73 \pm 0.92$ \\
\hline \multirow[t]{3}{*}{ Teaching philosophy } & \multirow[t]{3}{*}{$x^{2}=2.207(d f=2)$} & \multirow[t]{3}{*}{$p=0.332$} & Assigned & $3.15 \pm 1.05$ \\
\hline & & & Assigned and Selected & $3.56 \pm 0.73$ \\
\hline & & & Selected & $3.58 \pm 0.99$ \\
\hline \multirow[t]{3}{*}{ Teaching methods } & \multirow[t]{3}{*}{$x^{2}=5.613(d f=2)$} & \multirow[t]{3}{*}{$p=0.060$} & Assigned & $3.18 \pm 1.06$ \\
\hline & & & Assigned and Selected & $3.67 \pm 0.71$ \\
\hline & & & Selected & $3.81 \pm 0.94$ \\
\hline \multirow[t]{3}{*}{ Institutional hierarchy } & \multirow[t]{3}{*}{$X^{2}=5.437(d f=2)$} & \multirow[t]{3}{*}{$p=0.066$} & Assigned & $3.09 \pm 1.08$ \\
\hline & & & Assigned and Selected & $3.78 \pm 0.67$ \\
\hline & & & Selected & $3.62 \pm 0.75$ \\
\hline \multirow{3}{*}{$\begin{array}{l}\text { Service requirements (community, } \\
\text { profession, etc.)* }\end{array}$} & $x^{2}=7.067(d f=2)$ & $p=0.029$ & Assigned & $3.12 \pm 1.01$ \\
\hline & & & Assigned and Selected & $3.89 \pm 0.60$ \\
\hline & & & Selected & $3.65 \pm 0.75$ \\
\hline Institutional service requirements & $x^{2}=8.677(\mathrm{df}=2)$ & $p=0.013$ & Assigned & $3.06 \pm 0.98$ \\
\hline (comimillees, elc.) & & & Assigned and Selected & $3.89 \pm 0.60$ \\
\hline & & & Selected & $3.62 \pm 0.75$ \\
\hline Grant writing* & $x^{2}=9.943(d f=2)$ & $p=0.007$ & Assigned & $2.06 \pm 0.85$ \\
\hline & & & Assigned and Selected & $2.89 \pm 1.27$ \\
\hline & & & Selected & $2.85 \pm 0.97$ \\
\hline
\end{tabular}




\begin{tabular}{|c|c|c|c|c|}
\hline \multirow[t]{3}{*}{ Grant submission* } & \multirow[t]{3}{*}{$X^{2}=9.594(d f=2)$} & \multirow[t]{3}{*}{$p=0.008$} & Assigned & $2.06 \pm 0.85$ \\
\hline & & & Assigned and Selected & $2.78 \pm 1.39$ \\
\hline & & & Selected & $2.88 \pm 0.99$ \\
\hline \multirow[t]{3}{*}{ Advising* } & \multirow[t]{3}{*}{$x^{2}=6.141(\mathrm{df}=2)$} & \multirow[t]{3}{*}{$p=0.046$} & Assigned & $3.26 \pm 1.08$ \\
\hline & & & Assigned and Selected & $3.89 \pm 0.60$ \\
\hline & & & Selected & $3.85 \pm 0.88$ \\
\hline \multirow[t]{3}{*}{ Time commitment } & \multirow[t]{3}{*}{$X^{2}=0.425(d f=2)$} & \multirow[t]{3}{*}{$p=0.809$} & Assigned & $3.76 \pm 0.82$ \\
\hline & & & Assigned and Selected & $3.78 \pm 0.67$ \\
\hline & & & Selected & $3.92 \pm 0.80$ \\
\hline \multirow[t]{3}{*}{ Community engagement } & \multirow[t]{3}{*}{$X^{2}=2.532(\mathrm{df}=2)$} & \multirow[t]{3}{*}{$p=0.282$} & Assigned & $3.26 \pm 0.96$ \\
\hline & & & Assigned and Selected & $3.67 \pm 0.87$ \\
\hline & & & Selected & $3.62 \pm 0.98$ \\
\hline \multirow[t]{3}{*}{ Service learning } & \multirow{3}{*}{$X^{2}=0.345(d f=2)$} & \multirow[t]{3}{*}{$p=0.841$} & Assigned & $3.59 \pm 1.05$ \\
\hline & & & Assigned and Selected & $3.67 \pm 1.00$ \\
\hline & & & Selected & $3.46 \pm 0.95$ \\
\hline \multirow[t]{3}{*}{ Student professional guidance } & \multirow[t]{3}{*}{$x^{2}=0.136(d f=2)$} & \multirow[t]{3}{*}{$p=0.934$} & Assigned & $3.64 \pm 0.93$ \\
\hline & & & Assigned and Selected & $3.67 \pm 0.50$ \\
\hline & & & Selected & $3.73 \pm 0.87$ \\
\hline \multirow[t]{3}{*}{ Clinical and educational contracts } & \multirow[t]{3}{*}{$X^{2}=3.048(d f=2)$} & \multirow[t]{3}{*}{$p=0.218$} & Assigned & $3.12 \pm 1.23$ \\
\hline & & & Assigned and Selected & $3.00 \pm 1.12$ \\
\hline & & & Selected & $3.62 \pm 0.90$ \\
\hline \multirow[t]{3}{*}{ Creating a course } & \multirow[t]{3}{*}{$X^{2}=2.389(\mathrm{df}=2)$} & \multirow[t]{3}{*}{$p=0.303$} & Assigned & $3.32 \pm 1.09$ \\
\hline & & & Assigned and Selected & $3.56 \pm 0.53$ \\
\hline & & & Selected & $3.77 \pm 0.95$ \\
\hline \multirow[t]{3}{*}{ Creating syllabi } & \multirow[t]{3}{*}{$X^{2}=2.805(d f=2)$} & \multirow[t]{3}{*}{$p=0.246$} & Assigned & $3.41 \pm 1.10$ \\
\hline & & & Assigned and Selected & $3.56 \pm 0.53$ \\
\hline & & & Selected & $3.88 \pm 0.86$ \\
\hline Course/curricular sequencing & $X^{2}=3.597(d f=2)$ & $p=0.166$ & Assigned & $3.26 \pm 1.11$ \\
\hline & & & Assigned and Selected & $3.00 \pm 1.07$ \\
\hline & & & Selected & $3.73 \pm 0.83$ \\
\hline Socialization to academic culture* & $x^{2}=6.848(d f=2)$ & $p=0.033$ & Assigned & $3.24 \pm 1.02$ \\
\hline & & & Assigned and Selected & $3.56 \pm 0.73$ \\
\hline & & & Selected & $3.88 \pm 0.77$ \\
\hline $\begin{array}{l}\text { Expectations of institutional social } \\
\text { resnonsibilities }\end{array}$ & $X^{2}=5.643(d f=2)$ & $p=0.060$ & Assigned & $3.03 \pm 1.11$ \\
\hline & & & Assigned and Selected & $3.56 \pm 0.73$ \\
\hline & & & Selected & $3.58 \pm 0.90$ \\
\hline Collegiality and civility* & $\mathrm{X}^{2}=7.504(\mathrm{df}=2)$ & $p=0.023$ & Assigned & $3.24 \pm 1.12$ \\
\hline & & & Assigned and Selected & $4.22 \pm 0.83$ \\
\hline & & & Selected & $3.88 \pm 0.91$ \\
\hline
\end{tabular}

5-point Likert scale: 5=great knowledge, 4=more than average knowledge, 3=average knowledge, 2=little knowledge, 1=no knowledge at all * Significant differences at $p<0.05$ 


\section{Benefits of Mentorship}

Respondents were able to select from a list of benefits of mentorship and indicated that mentors assisted with publications $(n=21)$, presentations/posters $(n=27)$, new teaching methods/strategies $(n=45)$, clinical expertise $(n=19)$, conducting research $(n=20)$, service activities $(n=21)$, community engagement activities $(n=19)$, development of a program $(n=27)$, job change/promotion $(n=20)$, and grant writing/submissions $(n=11)$. In addition, respondents described their mentorship experiences as both positive and negative, dependent upon the mentor and circumstance (Table 5). The improvement over time indicated that those mentored gained knowledge about the professoriate and benefitted from mentorship.

Table 5. Characteristics of Mentorship Beyond Those Included in The Questionnaire Participant Statement

Due to my circumstances with my mentor leaving shortly after our "match" I am not sure I can really judge the mentorship program on campus or not. However, I wish I was re-assigned a mentor.

My mentor assigned to me was an undergraduate Music Department academic faculty member. The relationship was treated more as a forced friendship with little to no discussion about tenure, promotion, etc.

My mentor was great but I wish we had more formal, structured interactions rather than just me popping in when I had questions. I was overwhelmed and often didn't know the right questions to ask so I would have liked him to be more proactive.

He got me started but almost all those items on your scaled I learned as I went along

Since my mentor was not in my field, there was little assistance I thought she could provide in my first year. My major challenges that year were putting together a self-study and site visit without a program director. I didn't have time to worry about everything else.

Mentors can be very helpful. The mentors I had at the time I wouldn't call them mentors, just advisors who were interested and available. After leaving them 6 years ago, I realized they were really mentors.

I found different experienced academic faculty members to mentor me in their area of expertise as needed. I had already been published and given presentations but one of my mentors encouraged me to present at conferences that I would not have considered otherwise.

I responded based on the mentor I was assigned by my institution as a new academic faculty member. I consider other colleagues mentors as well. They have had a much larger impact on my career and my understanding of the professoriate.

I was assigned a mentor who was essentially available for questions, but had no guided interactions in any way.

My mentor "walked the walk" and "talked the talk."

My interactions with my assigned "mentor" were really no different than my interactions with anyone else on the academic faculty. Little to no guidance was provided and most new information I stumbled upon on my own.

I have two mentors, one of whom I have met with twice and the other for research guidance only once. Most issues have not been discussed.

She values me as a person, beyond my professional expertise and skills as an educator.

\section{DISCUSSION}

Prior research indicated that mentorship in academia, particularly health professions increases productivity, promotions, employment satisfaction, and academic productivity. ${ }^{16-23}$ Mentorship and mentorship programs are a combination of intellectual information and emotional connection creating a foundation of trust and support that is projected into junior academic faculty action and growth. ${ }^{25}$ Our findings confirm the literature that emphasizes the benefit of mentorship relationships. We identified that academic faculty who were mentored had more positive experiences than academic faculty who were not mentored, which may translate into increased retention and productivity. ${ }^{16-23}$ More mature academic faculty will benefit students. From an institutional 
perspective, academic faculty mentorship is essential for retention, production, and educational continuity in order to better serve students and promote excellence in education. ${ }^{3}$

The love for learning and a desire to interact with an intellectual community is often what motivates individuals to pursue a career in academia. ${ }^{14}$ Yet junior academic faculty must often make personal sacrifices they did not intend in a political environment they did not foresee, which can emphasize vague expectations for academic life and therefore increase dissatisfaction. ${ }^{15-22}$ Mentorship appears to attenuate the challenges junior academic faculty face in navigating faculty requirements and expectations. ${ }^{1-9,23}$ Our current study is congruent with the literature in that academic faculty who are mentored perceive they have a better understanding of the academic requirements (specifically scholarship). Interestingly, among mentored individuals, institutional hierarchy, service, community engagement, and teaching methods were areas of mentorship where junior academic faculty felt less engaged. This finding is concerning given that the incongruity between junior academic faculty expectations and academic requirements are what diminishes optimism, eagerness, and job satisfaction over time. ${ }^{17}$ Although mentored academic faculty feel supported in scholarship (publications, presentations, and grants), an understanding of academic culture is essential to mitigate the disconnect between true and perceived expectations of full-time academic faculty duties. A large component of transitioning is the culture assimilation to higher education, beyond scholarship, that includes further understanding of institutional policies, hierarchy, service, and community engagement expectations as well as teaching methods. Individuals who lack a mentor to guide them in all areas of academia are more at risk for isolation, do not feel as closely tied to their department or institution, and may rely on risky strategies of trial and error to build their academic and institutional careers. ${ }^{18,25-27}$ These behaviors may jeopardize student education, potentially deteriorating the department/college work environment as well as perceptions of program/academic faculty effectiveness.

Educators with clinical doctorates who are trained to focus on clinical practice may have an exacerbated need for acculturation and mentorship because many are not educated in the areas of scholarship or teaching while many traditionally educated academic faculty $(\mathrm{PhD} / \mathrm{EdD})$ have those experiences. Health professions education is unique compared to traditional higher education with a larger number of academic faculty holding clinical doctorates as opposed to traditional terminal degrees

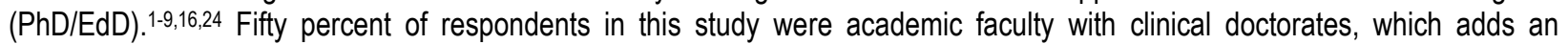
additional layer of mentorship needs. Transition from doctoral preparation or clinical experience to academia is a stressor for most new academic faculty and successes are often measured through the tenure and promotion process which may be elusive to clinically trained academic faculty. 1,2 Compounding the lack of understanding for academic faculty requirements may be issues of decoding expectations of the organization and a lack of pedagogical skill. ${ }^{16,20}$ Mentorship must be both a planned and informal emotional interaction in order to effectively direct and support all junior academic faculty, with some specific focus on clinically trained academic faculty who may have less understanding of the requirements. ${ }^{25}$

We found that academic faculty who received mentorship were primarily assigned a mentor, but others also sought out individuals they felt would help them navigate their new position. Overall, individuals whose mentorship experience was a combination of assigned and self-selected mentors perceived more benefits from the mentorship relationship. Literature also suggests that most new academic faculty who are mentored are assigned more mature academic faculty, indicating that administrators or academic faculty do feel a sense of responsibility to new colleagues. ${ }^{17}$ These relationships provide the foundation of security and acceptance and allow new academic faculty to reach out to other mature academic faculty members for their needs. In addition to assigned mentors, institutions should provide a planned mentorship program that addresses organizational hierarchy, service, and community engagement requirements. Similar to published literature, we identified that most mentoring centers around scholarship, whereas dissatisfaction is primarily related to a lack of understanding of the social and institutional expectations. Although literature on forced mentorship is variable, our findings juxtaposed against the literature suggests that an initial mentorship assignment benefits academic faculty and institutions particularly because approximately a third of academic faculty have indicated that they cannot find a suitable mentor willing to assist in professional development. ${ }^{17,24,26-28}$

Socialization during traditional doctoral preparation may lead junior academic faculty to develop unrealistic expectations regarding faculty collegiality, research, and responsibility. ${ }^{29}$ Traditional doctoral trained faculty may not participate in institutional hierarchy, service, or advising/administrative responsibilities that provide a disproportionate view of time management allotted for research and teaching. ${ }^{29}$ Likewise, clinical faculty who may not have any orientation to faculty responsibilities may have misconceptions of the job expectations. ${ }^{29}$ Institutional and academic faculty acculturation is necessary to orient new academic faculty, reduce dissatisfaction and increase talent retention. The current study suggests that approximately three quarters of junior academic faculty perceive that they did not receive any direct mentoring by other academic faculty, administrators, or programs. Literature also articulates that a direct correlation between mentorship and satisfaction exists, and that junior academic faculty who are mentored have increased retention, salary, production, and overall satisfaction. ${ }^{17-24}$

(c) The Internet Journal of Allied Health Sciences and Practice, 2013 
Mentorship, whether one-on-one or in planned group environments, should include a multitude of components of scholarship, teaching, and institutional information (Figure 1). Mentoring new academic faculty may take many forms including one-on-one engagement, peer group mentoring, formal planned mentoring by the institution, social events, and informal relationships. 8-13,25,29 Regardless of mentoring method, to effectively impact and socialize new academic faculty, common principles must exist which include an environment of trust, security, and transfer of information..$^{25,29}$ Institutions must evaluate the mentorship models that will best fit their resources and culture.

\section{LIMITATIONS}

Although all academic faculty employed in the United States were surveyed, the representation of respondents is not equal which may affect generalization to, specifically, PT faculty $(n=3)$. In addition, medical academic faculty was not assessed and should be investigated to determine whether parallel themes exist in that academic structure.

\section{Figure 1. Components of Mentorship}

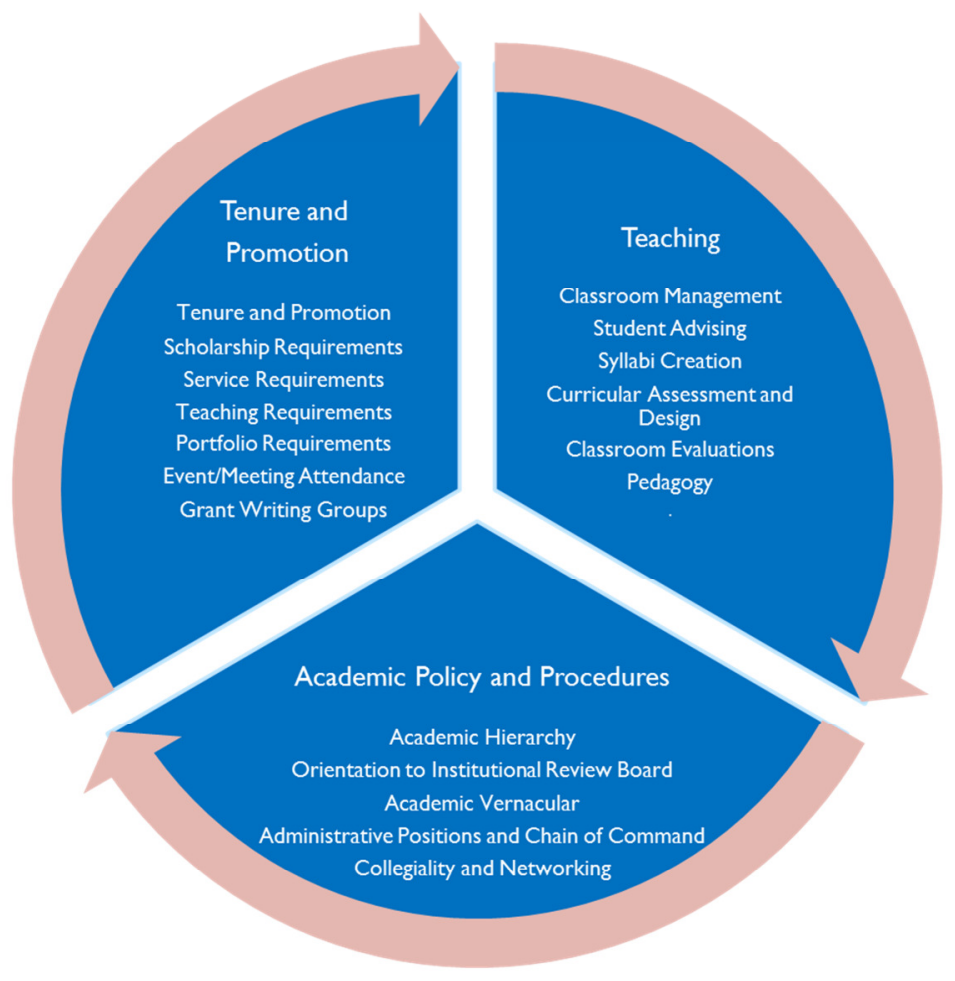

\section{CONCLUSIONS}

New academic faculty are more sensitive to workplace stressors, and medical/health care academic faculty are no different, other than the potential added clinical responsibility. A lack of time, work overload, and high self-esteem expectations are components of decreased satisfaction and burnout among junior academic faculty. Junior academic faculty concerns are in large part due to incongruity between the expectations articulated (or not articulated) during doctoral studies or clinical experiences and the actual requirements of a full-time academic faculty member. Our study indicates that academic faculty who were mentored had a more positive experience and perceived they had a better understanding of the academic environment with respect to scholarship and teaching. However, institutional hierarchy/policies, service, and community engagement are areas of mentorship that need to be expounded in order to protect academic faculty, institutions, and students from dissatisfaction and associated outcomes of mismatched expectations. Likewise, academic faculty that do not receive mentorship, which was $75 \%$ in this study, are more likely to disassociate, practice risky career behaviors, and negatively impact the culture and work environment of the institution. Mentorship programs for new academic faculty are essential to facilitate the growth of the individual and institution. Multiple models for mentorship programs are available and should be reviewed and implemented based on institutional culture, resources, and available mentors. 


\section{REFERENCES}

1. Olson D. Work satisfaction and stress in the first and third year of academic appointment. J High Educ. 1993;64:453-71.

2. Sorcinelli MD, Billings DA. The career development of pretenure academic faculty: An institutional study. Annual Meeting of the American Educational Research Association. Atlanta, GA, April 12-16. 1993.

3. Smith DL. Perceptions by practicing occupational therapists of the clinical doctorate in occupational therapy. Journal of Allied Health. 2007;36(3):137-40.

4. Bieber JP, Worley Lk. Conceptualizing the academic life: Graduate students' perspectives. J Higher Ed. 2006;77(6):100935.

5. Austin AE. Preparing the next generation of academic faculty: Graduate school as socialization to the academic career. $J$ Higher Ed. 2002;73(1):94-122.

6. Golde CM, Dore TM. At cross purposes: What the experiences of today's doctoral students reveal about doctoral education. http://www.phd-survey.org/report\%20final.pdf Accessed January 10, 2013

7. Adams KA. What colleges and universities want in new academic faculty. Washington, DC: Association of American Colleges and Universities:2002. www.aacu.org/pff/pdfs/pff_adams.pdf Accessed January 10, 2013.

8. American Association of Colleges of Nursing. 2010. DNP Fact Sheet. http://www.aacn.nche.edu/dnp Accessed January 10, 2013.

9. Royeen C, Lavin MA. A contextual and logical analysis of the clinical doctorate for health practitioners: dilemma, delusion, or de facto? Journal of Allied Health. 2007;36(2):101-6.

10. Smith DL. Preceptions by practicing occupational therapists of the clinical doctorate in occupational therapy. Journal of Allied Health. 2007;36(3):137-40.

11. Dickerson AE, Trujillo L. Practitioners' perceptions of the occupational therapy clinical doctorate. Journal of Allied Health. 2009;38(1):e47-53.

12. Eisenhauer L, Bleich MR. The clinical doctorate: Whoa or go? J Nurs Educ. 2006;45(1):3-4. [PMID 16496731]

13. Senft SL, Thompson C, Blumenschein K. Dual degree programs at the University of Kentucky College of Pharmacy. . Am J Pharm Educ. 2008;72(1):12. [PMID 18322574]

14. Kelley KA, Coyle JD, McAuley JW, Wallace LJ, Buerki RA, Frank SG. Writing PharmD program-level, ability-based outcomes: key elements for success. Am J Pharm Educ. 2008;72(5):98. [PMID 19214252]

15. Montoya ID, Kimball OM. Marketing clinical doctorate programs. J Allied Health. 2007 Summer;36(2):107-12. [PMID 17633968]

16. Schrodt $P$, Cawyer CS, Sanders R. An examination of academic mentoring behaviors and new academic faculty members 'satisfaction with socialization and tenure and promotion processes. Commun Educ. 2003;52:17-29.

17. Feldman M. Does mentoring matter: results from a survey of academic faculty mentees at a large health sciences university. Med Ed Online. 2010:15, www.ncbi.nlm.nih.gov/pmc/articles/PMC2860862/ Accessed January 10, 2013.

18. Alexander JC. Mentoring: On the road to tenure and promotion. ACA Bulletin. 1992;79;54-8.

19. Kram KE. Mentoring at Work: Developmental Relationships in Organizational Life. Glenview, IL: Scott, Foresman, 1985.

20. Sambunjak D, Straus SE, Marusic A. Mentoring in academic medicine: a systematic review. JAMA. 2006;296(9):1103-15. [PMID 16954490]

21. Garman KA, Wingard DL, Reznik V. Development of junior academic faculty's self-efficacy: outcomes of a national center of leadership in academic medicine. Acad Med. 2001;76(10):S74-6. [PMID 11597879]

22. Ramanan RA, Phillips RS, Davis RB, Silen W, Reede JY. Mentoring in medicine: keys to satisfaction. Am J Med. 2002;112(4):336-41. [PMID 11893387]

23. Steiner JF, Lanphear BP, Curtis $\mathrm{P}$, Vu KO. Indicators of early research productivity among primary care fellows. J Gen Intern Med. 2002;17(11):845-51. [PMID 12406356]

24. Pololi L. Career development for academic medicine - a nine step strategy. BMJ Careers. 2006.

25. Kahanov L, Eberman LE, Yoder A, Kahanov M. Culture Shock: transitioning from clinical practice to educator. Int J Allied Health Sci Prac. 2012;10(1).

26. Wingard DL, Garman KA, Reznik V. Facilitating academic staff/faculty success: outcomes and cost benefit of the UCSD National Center of Leadership in Academic Medicine. Acad Med. 2004;79(10 Suppl):S9-11. [PMID 15383376]

27. Carr P, Bickel J, Inui TS, editors. Taking root in a forest clearing: a resource guide for medical academic faculty. Boston, MA: Boston University School of Medicine and the W.K. Kellogg Foundation; 2003.

28. Kalet AL, Fletcher KE, Ferdman DJ, Bickell NA. Defining, navigating and negotiating success: the experienced of midcareer Robert Wood Johnson Clinical Scholar women. J Gen Intern Med. 2006;21(9):920-5. [PMID 16918735]

29. Eberman L, Kahanov L. Mentorship of New Academic faculty Members. Int J Ath Ther Train. 2011;16(60):34-7. 